\title{
Correlation between Doppler Indices as A Predictor of Neonatal Outcome in Normal and Preeclamptic Pregnant Women in The Third Trimester
}

Abdel-Hamid E. Shaheen, Mohamed A. Elsayed, Ragab M. Dawood, Hoda R. Abbas

Obstetrics and Gynecology Department, Faculty of Medicine, Menoufia University, Menoufia, Egypt

*Corresponding author: Hoda Ramadan Ahmed Abbas, Mobile: (+20)01205268505, E-mail: hodaelsaqa@ yahoo.com

\begin{abstract}
Background: Doppler flow velocimetry of the umbilical artery and cerebral circulation is a non-invasive modality used for assessment of fetal wellbeing. Doppler studies are more specific and are potentially useful tool in prediction of adverse perinatal outcome in high risk patients. Aim of the current study was to evaluate the efficacy of middle cerebral artery pulsatility index (MCA-PI), umbilical artery pulsatility index (UA-PI), uterine artery pulsatility index (UAT-PI), and a cerebroplacental ratio (CPR) Doppler indices in assessment of fetal wellbeing. For documentation of the neonatal outcome in preeclamptic women with abnormalities of Doppler indices.

Objectives: Assessment of fetal wellbeing and prediction of neonatal outcome in normal pregnant women and woman with preeclampsia by using Doppler indices.

Patients and Methods: the study is a case control study included 94 pregnant women (47 normal pregnancy and 47 preeclamptic patients) admitted in Obstetrics and Gynecology Department of Menoufia University during the period from August 2018 to November 2019. All included women underwent Doppler study and were followed up till delivery. Results: Doppler velocimetry studies of both placental and fetal circulation can provide important information as regard fetal wellbeing so can be used to improve fetal outcome.

Conclusions: It was observed that all three parameters UAT-PI, MCA-PI and UA-PI when taken into consideration together are good parameters in predictions of perinatal outcome.
\end{abstract}

Keywords: Doppler, Umbilical artery, Preeclampsia.

\section{INTRODUCTION}

Pregnancy induced hypertension is one of the most important causes of maternal and perinatal morbidity and mortality in developing countries. As it leads to abnormal placentation, which involves the abnormal trophoblast invasion of spiral arteries resulting in an increase in vascular resistance of the uteroplacental circulation. This process also may cause intrauterine growth restriction (IUGR) in the absence of adequate management ${ }^{(\mathbf{1})}$.

In cases with placental insufficiency, the fetal circulation undergoes numerous changes, ending in brain sparing effect. Brain sparing phenomena is caused by redistribution of blood flow to most important organs like the brain, heart and adrenals at the expense of other organs such as spleen, kidney and peripheral circulation ${ }^{(2)}$.

Assessment of Doppler flow velocimetry of the umbilical and fetal cerebral circulation is a noninvasive modality used to assess the fetal wellbeing. Doppler is more specific and is potentially a useful tool used in the in prediction of adverse perinatal outcome in high risk cases ${ }^{(3)}$. Cerebroplacental (CP) ratio has already been very established predictor of adverse pregnancy outcome. The cerebroplacental ratio can be used in detection of fetal response to oxygen deficiency in utero, the values of resistance index of middle cerebral artery (MCA RI) should remain higher than umbilical artery resistance ratio (UA RI) values, which implicates that cerebroplacental ratio, being the ratio of MCA RI to UA RI, should be higher than 1-1.1 in low risk pregnancies ${ }^{(4)}$.
Thus an attempt is made to evaluate the efficacy of middle cerebral artery pulsatility index (MCA-PI) umbilical artery pulsatility index (UA-PI), uterine artery pulsatility index (UAT-PI) and cerebroplacental ratio (CPR) in term women with preeclampsia and fetal outcome.

\section{PATIENTS AND METHODS}

Case control study was conducted in Department of Obstetrics and Gynecology, Faculty of Medicine, Menoufia University and Menuf General Hospital, Menoufia Governorate Egypt.

\section{Ethical approval}

Informed consent was taken from all participants following approval of the Ethical Committee of both institutes of Menoufia University.

Recruitment of cases started 1 August 2018 till November 2019. 94 pregnant women were involved in the study and divided into 47 normal pregnant women and 47 pregnant preeclamptic patients (mild-severe).

All patients were subjected to thorough history taking, clinical evaluation with on full medical and surgical history. The clinical examination included general examination (height, weight, vital data) and abdominal examination (inspection, size of the abdomen). The laboratory investigation were as usual in the Department of Obstetrics and Gynecology in the hospital for all cases (CBC, urine analysis, liver function tests, kidney function tests, serum uric acid level, fasting and 2 hours postprandial blood glucose level and HBA1c). 
All the cases underwent the routine obstetric scan to know the fetal biometry to assess gestational age, fetal weight, placental age and Doppler indices, which are MCA-PI, UA-PI, UAT-PI and CPR was done. These values were considered abnormal when they would lie below the $5^{\text {th }}$ percentile and above the $95^{\text {th }}$ percentile. Perinatal outcome was documented. The adverse outcomes like cesarean section for fetal distress, meconium-stained liquor, 5 minute Apgar $<7$, IUGR (fetal weight $<10^{\text {th }}$ for gestational age) babies requiring NICU admission for more than $24 \mathrm{~h}$ or preinatal death.

\section{Statistical analysis}

Data were verified, coded by the researcher and analyzed using IBM-SPSS for windows, version 23.0 (Copyright IBM Corp., Armonk, N.Y., USA, 2015). Descriptive statistics: Means, standard deviations, medians, ranges and percentages were calculated. Chi- square test was used to compare the difference in distribution of frequencies among different groups. For continuous variables; independent t-test analysis was carried out to compare the means of quantitative data. There was no specific calculation of the sample size. A significant $\mathrm{p}$-value was considered when it is equal or less than 0.05 .

\section{RESULTS}

A total 47 preeclamptic women, $8.5 \%$ of women had normal delivery and $91.5 \%$ had lower segment cesarean section. $97.9 \%$ of delivered babies required NICU (neonatal ICU) care. 95.7\% of delivered babies had Apgar score $<7$ in first 5 minutes. The perinatal complications like (low birth weight, meconium stained, still born and perinatal asphyxia) were in 95.7\%. There was significant decrease MCA/umbilical artery PI and decrease MCA/uterine artery PI in preeclamptic group (Table 1).

Table (1): Comparison between the two studied groups according to MCA/UA PI AND MCA/UAT PI

\begin{tabular}{|l|c|c|c|c|}
\hline \multicolumn{1}{|c|}{ PI } & Control $(\mathbf{n}=\mathbf{4 7})$ & Patients $(\mathbf{n}=\mathbf{4 7})$ & $\mathbf{U}$ & p \\
\hline MCA/UA ratio (PI) & & & & \\
Min.-Max. & $0.99-2.66$ & $0.01-2.63$ & & \\
Mean \pm SD. & $2.29 \pm 0.25$ & $1.19 \pm 0.57$ & 111.5 & $<0.001^{*}$ \\
Median & 2.29 & 0.96 & & \\
\hline MCA/UAT (PI) & & & & \\
Min.-Max. & $0.91-2.67$ & $0.84-2.28$ & & \\
Mean \pm SD. & $2.0 \pm 0.45$ & $1.16 \pm 0.41$ & 291.50 & \\
Median & 2.02 & 0.98 & & \\
\hline
\end{tabular}

U: Mann Whitney test, *: Statistically significant

There was significant increase rate of caesarean section and decreased normal vaginal delivery rate in preeclamptic group (Table 2).

Table (2): Comparison between the two studied groups according to mode of delivery and IUGR

\begin{tabular}{|c|c|c|c|c|c|c|}
\hline & \multicolumn{2}{|c|}{ Control $(n=47)$} & \multicolumn{2}{|c|}{ Patients $(n=47)$} & \multirow[t]{2}{*}{ Test of Sig. } & \multirow[t]{2}{*}{$\mathbf{p}$} \\
\hline & No. & $\%$ & No. & $\%$ & & \\
\hline \multicolumn{7}{|l|}{ Mode of delivery } \\
\hline $\mathrm{C} / \mathrm{S}$ & 13 & 27.7 & 43 & 91.5 & \multirow{2}{*}{$\chi^{2}=39.756$} & \multirow{2}{*}{$<0.001^{*}$} \\
\hline NVD & 34 & 72.3 & 4 & 8.5 & & \\
\hline IUGR & \multirow{2}{*}{\multicolumn{2}{|c|}{$2100-3850$}} & \multirow{2}{*}{\multicolumn{2}{|c|}{$2100-3100$}} & \multirow{4}{*}{$\mathrm{t}=9.534$} & \multirow{4}{*}{$<0.001^{*}$} \\
\hline Min-Max & & & & & & \\
\hline Mean \pm SD & \multicolumn{2}{|c|}{$3146 \pm 338.3$} & \multicolumn{2}{|c|}{$2536.6 \pm 278.6$} & & \\
\hline Median & \multicolumn{2}{|c|}{3200.0} & \multicolumn{2}{|c|}{2600.0} & & \\
\hline
\end{tabular}

$\mathrm{c}^{2}$ : Chi square test t: Student t-test, *: Statistically significant

Figure 1 shows significant decrease of fetal Apgar score (A/S) of preeclamptic patient. 


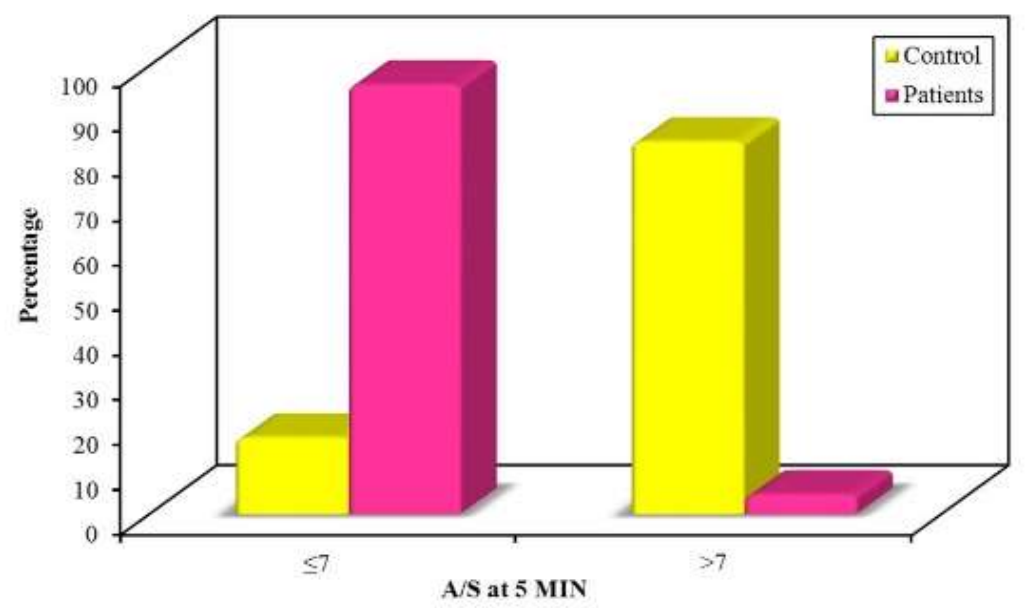

Figure (1): Comparison between the two studied groups according to A/S at 5 minutes

There is significant increase the rate of NICU admission of babies of preeclamptic patients (Table 3).

Table (3): Comparison between the two studied groups according to NICU admission

\begin{tabular}{|c|c|c|c|c|c|c|}
\hline \multirow{2}{*}{$\begin{array}{c}\text { NICU } \\
\text { admission }\end{array}$} & \multicolumn{2}{|c|}{$\begin{array}{l}\text { Control } \\
(n=47)\end{array}$} & \multicolumn{2}{|c|}{$\begin{array}{r}\text { Patients } \\
(n=47)\end{array}$} & \multirow[t]{2}{*}{ Test of Sig. } & \multirow[t]{2}{*}{$\mathbf{p}$} \\
\hline & No. & $\%$ & No. & $\%$ & & \\
\hline No & 36 & 76.6 & 1 & 2.1 & \multirow{2}{*}{$\begin{array}{c}\chi^{2}= \\
54.599^{*}\end{array}$} & \multirow{2}{*}{$<0.001^{*}$} \\
\hline Yes & 11 & 23.4 & 46 & 97.9 & & \\
\hline Min. - Max. & \multicolumn{2}{|c|}{$0.25-2.0$} & \multicolumn{2}{|c|}{$0.25-8.0$} & \multirow{3}{*}{$\begin{array}{c}\mathrm{U}= \\
102.5^{*}\end{array}$} & \multirow{3}{*}{$0.002^{*}$} \\
\hline Mean \pm SD & \multicolumn{2}{|c|}{$0.95 \pm 0.56$} & \multicolumn{2}{|c|}{$2.39 \pm 1.62$} & & \\
\hline Median & \multicolumn{2}{|c|}{1.0} & \multicolumn{2}{|c|}{2.0} & & \\
\hline
\end{tabular}

$c^{2}$ : Chi square test U: Mann Whitney test

*: Statistically significant

There was statistically significant difference of fetal maturity between control and patient groups (Figure 2).

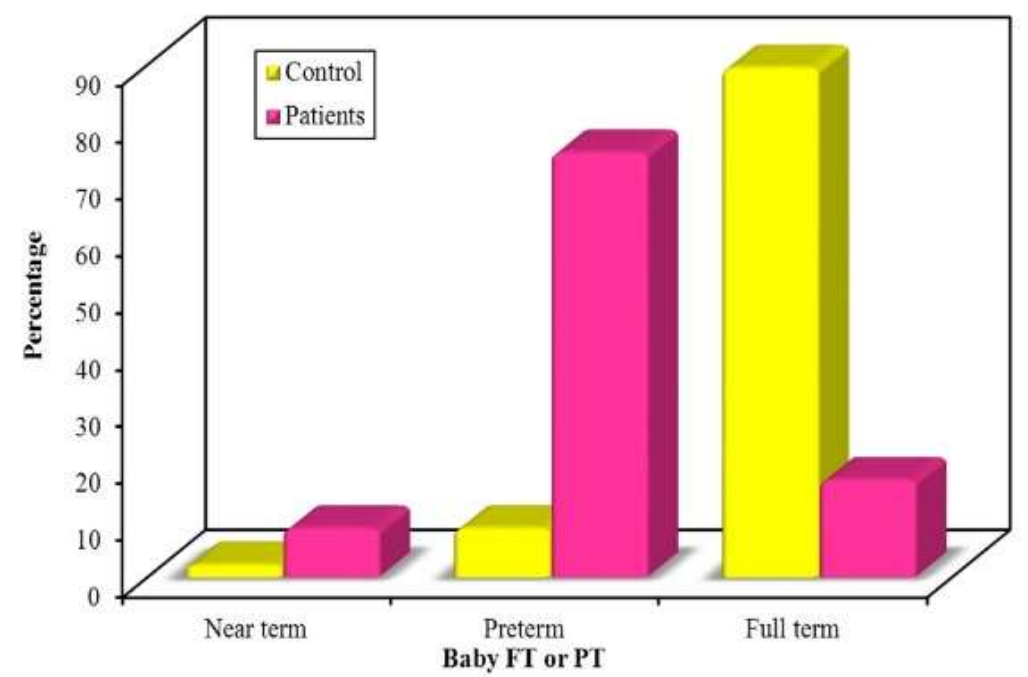

Figure (2): Comparison between the two studied groups according to baby FT or PT.

\section{DISCUSSION}

The present study was done to detect the relation between Doppler indices and neonatal outcome in normal pregnancy. It also evaluated the relation between Doppler indices and neonatal outcome in preeclampsia complicated pregnancy. 
The current study revealed that, there was no statistically significant difference between the two groups as regard maternal age. This is comparable to study done by Adekanmi et al. ${ }^{(5)}$ who also found no significant difference in the age between the two groups $(\mathrm{P}=0.936)$. The mean age of pregnant women who developed preeclampsia (PE) was $32.03 \pm 4.11$ years with a range of 22-39 years, while the mean age of pregnant women who did not develop PE was $31.65 \pm 6.42$ years with a range of $15-48$. Also, the results were close to that done by Raj and Sheela ${ }^{(6)}$ who found the mean of age was $25.13 \pm 4.33$ years, minimum age was 18 years and maximum age was 36 years. The results of the present study were disagreed with Liu et al. ${ }^{(7)}$ who reported the age, which showed significant difference among eclampsia cases and controls. The difference may be due to the patient selection criteria of preeclampsia cases employed in their study compared with PE cases in the present study.

In the current study, it a significant decrease in platelets count among patients than controls was found while, no significant difference was found between the studied groups regarding HB and HCT. This is consistent with results of studies done by Ahmed et al. ${ }^{(8)}$ and Järemo et al. ${ }^{(9)}$ that revealed lower platelets count in preeclamptics; referring these changes to increased platelet consumption in preeclampsia. Also, Makuyana et al. ${ }^{(10)}$ found no difference in hemoglobin levels between preeclamptic and controls. Additionally, the results of a study done by $\mathbf{1 1}$. El

Sherbiny et al. ${ }^{(11)}$ reported no significant difference in hemoglobin level and hematocrit value between pregnant women with preeclampsia (mild and severe preeclampsia) and their matched control group. While the results of a study done by El-garhey et al. ${ }^{\left({ }^{12}\right)}$ found no statistically significant difference according to HB level and platelet count when preeclamptic patients were compared with controls. Also, in the study done by Makuyana $\boldsymbol{e t}$ al. ${ }^{(10)}$ they found no difference in PLTs count between preeclamptic and controls. In addition, Fay et al. $^{(\mathbf{1 3})}$ found that there was a decrease in platelet count throughout the normal pregnancy reaching 30 weeks and suggested that the decrease was due to plasma dilution and increased consumption

The study showed a significant increase among patients' group than controls as regard systolic and diastolic pressures. This was consistent with Eser $\boldsymbol{e t}$ al. ${ }^{(14)}$. Also, with Liu et al.'s ${ }^{(7)}$ report in which systolic and DBP showed significant difference among preeclampsia cases and controls.

From the results of the present study it was revealed that, there was a statistically significant increase among patients than controls regarding Doppler indices of UA and UTA (RI and PI). This was comparable to, Adekanmi et al. ${ }^{(5)}$ who observed increased impedance indices (RI, PI and S/D) in both the uterine and umbilical arteries in women that developed PE compared with those that did not. In line with our study, the significantly higher mean RI and PI among PE cases compared to women without PE, support the report by Mallikarjunappa et al. ${ }^{(15)}$ that the uterine and umbilical artery Doppler study showed elevation of these two parameters among pregnant women with PE in the second and third trimester. This was also corroborated by Li et al.'s ${ }^{(16)}$ report.

In comparison to, Lopez-Mendez et al. ${ }^{(1)}$; it was observed that there was no significant difference in the uterine arteries' PI and RI, but a significant difference in the umbilical artery PI and RI between women with PE and those without PE, which is similar to our findings in the umbilical arteries in the current study. Also, Özeren et al. ${ }^{(17)}$ demonstrated that there was no difference between normal pregnancies and preeclamptic found that patients without IUGR regarding mean umbilical artery Doppler values. The difference between results of either studies could be because of differences in patients Doppler time and the abnormality limits of obstetric Doppler parameters, which may differ between populations.

This study showed that, there was a statistically significant decrease in Doppler MCA (S/D, $\mathrm{RI}$ and PI) among patients' group than controls. This agreed with Özeren et al. ${ }^{(17)}$ who showed that MCA PI value was lower in the preeclamptic groups. Also, Abdelrazik et al. ${ }^{(18)}$ found that the fetuses with adverse outcome had a lower middle cerebral artery PI index, supporting the finding of Brar et al. ${ }^{(19)}$ and Arduini and Rizzo ${ }^{(20)}$.

This study revealed that, MCA/UA PI and MCA/UAT PI ratio was significantly decreased among preeclamptic than the control group. This agreed with Ghosh et al. ${ }^{(21)}$ who found that MCA PI/UA PI ratio was $<1$, which was statistically significant $(\mathrm{p}<0.05)$ in $25.5 \%$ and absent in $17.3 \%$ among cases of adverse perinatal outcome. Also Shahinaj et al. ${ }^{(22)}$ found high sensitivity of the MCA/UA PI ratio in predicting stillbirth (100\%). Also, they found a high specificity and positive predicting value in need for treatment in neonatal intensive care unit. The author found that UA/MCA PI index ratio was the best test when compared with MCA, UA, and RA PI indices (sensitivity 89\%, specificity 94\%). Their results suggested that the MCA/UA PI Doppler ratio of less than 1 was a good predictive tool for neonatal outcome in preeclamptic and hypertensive pregnant women and could be used to identify fetuses at risk of morbidity and mortality. According to Arduini and Rizzo ${ }^{(20)}$ and Gramellini et al. ${ }^{(23)}$ assessment of MCA/UA PI index provide better information in predicting perinatal outcome when compared with umbilical or middle cerebral artery Doppler indices alone.

The results of the present study showed that there were statistically significant difference between 
patients and controls as regard Apgar score in first 5 minutes of fetal outcome. Patients had lower Apgar score than controls, in addition to most of them had NICU admission. This is consistent with Ayaz et al. ${ }^{\text {(24) }}$ who demonstrated that, low Apgar score was seen in cases as compared to controls ${ }^{(25)}$ and there was high need for NICU admission (26.02\%) in cases compared to controls (9.5\%). Abdelrazik et al. ${ }^{(18)}$ also revealed that the group of MCA/UA PI ratio <1 was significantly associated with 5-minute Apgar score less than 7. The result of this study is comparable to those of El-Sokkary et al. (26), which found that the rate Apgar score $<7$ was higher with MCA/UA PI ratio $<1$. They also found that MCA PI/UA PI ratio showed a $73.7 \%$ sensitivity and $68.3 \%$ specificity and a $52 \%$ positive predictive value and $85 \%$ negative predictive value in prediction of prenatal outcome (Apgar score $<7)$ and were higher with MCA PI/UA PI <1.

In the results of the study done by Abdelrazik et al. ${ }^{(18)}$ it was found that the MCA/UA PI ratio had $62.5 \%$ sensitivity and $71.43 \%$ specificity as predictors of NICU admission, with cut off value 29.41 positive predictive values and negative predictive value 90.91 . They also showed that the group of MCA/UA PI ratio $<1$ was significantly associated with higher rate of neonatal ICU (62.5\%). Eser et al. ${ }^{(14)}$ found that NICU admission was ( 26.3 vs. $57.6 \%$ ). Additionally, Yalti $\boldsymbol{e t}$ al. (27) revealed that fetus with abnormal Doppler velocimetry had a significantly higher admission to NICU.

In the current study it was revealed that there was a statistically significant increase in patients as regard the mode of delivery, presence of IUGR and complications. Most patients had C.S, higher IUGR and complications. This is comparable to results of the study done by Abdelrazik $\boldsymbol{e t}$ al. ${ }^{(18)}$, which found that IUGR was significantly higher than controls. Different post-natal complications were found in babies of complicated case with jaundice, sepsis, need for antibiotics and hypoglycemia. Causes of early neonatal deaths in cases were hypoglycemia, hematemesis and early neonatal sepsis, while causes of early neonatal death in controls were found to occur as a result of meconium aspiration ${ }^{(28)}$.

Also, in a study done by Shwarzman et al. ${ }^{(29)}$, which was a prospective study done to assess the correlation between abnormal uterine artery resistance in the third trimester of pregnancy and pregnancy outcomes, it was observed that a higher rate of cesarean delivery and preterm deliveries, small-for-gestationalage neonates, and lower Apgar scores in neonates born to women with bilateral pathologic uterine artery Doppler waveforms than in those with normal or pathologic unilateral waveforms. Thus, they concluded that abnormalities in uterine artery Doppler velocimetry detected in the late trimester are a good predictor of adverse perinatal outcomes of low and high risk pregnancy. In the study that was done by Ayaz et $\boldsymbol{a l}{ }^{(24)}$ it was found that IUGR was significantly present in cases.

\section{CONCLUSION}

There is a strong correlation between the MCA/UA (PI) and MCA/ UT (PI) ratio and neonatal outcome; it was revealed that a normal MCA/UA (PI) and MCA/uterine (PI) ratio is a strong predictor of a normal fetal outcome, while an abnormal values is strongly associated with neonatal morbidity.

It was recommended that further studies to be done on a larger scale, with introduction of new Doppler measurements to different fetal vessels, and to measure different indices, which might be useful in prediction of adverse neonatal outcome in pregnant women with preeclampsia.

\section{REFERENCES}

1. Lopez-Mendez M, Martinez-Gaytan V, Cortes-Flores R et al. (2013): Doppler ultrasound evaluation in preeclampsia. BMC Res Notes, 6 (1):477-83.

2. Adiga P, Kantharaja I, Hebbar S et al. (2015): Predictive value of middle cerebral artery to uterine artery pulsatility index ratio in hypertensive disorders of pregnancy. Int J Reprod Med., 1:5-8.

3. Ropacka-Lesiak M, Korbelak T, Świder-Musielak J et al. (2015): Cerebroplacental ratio in prediction of adverse perinatal outcome and fetal heart rate disturbances in uncomplicated pregnancy at 40 weeks and beyond. Arch Med Sci., 11 (1):142-8.

4. Mirghani $\mathrm{H}$, Weerasinghe $\mathrm{D}$, Ezimokhai $\mathrm{M}$ et al. (2003): The effect of maternal fasting on the fetal biophysical profile. Int J Gynaecol Obstet., 81: 17-21.

5. Adekanmi A, Roberts A, Akinmoladun J et al. (2019): Uterine and umbilical artery doppler in women with preeclampsia and their pregnancy outcomes. Nigerian Postgraduate Medical Journal, 26 (2):106-9.

6. Raj K, Sheela S (2020): The efficacy of Doppler indices in predicting the neontal outcome in term preeclamptic women with intrauterine growth restriction: an observational study in a tertiary care centre. International Journal of Reproduction, Contraception, Obstetrics and Gynecology, 9(5):2088-93.

7. Liu C, Cheng P, Chang S et al. (2008): Maternal complications and perinatal outcomes associated with gestational hypertension and severe preeclampsia in Taiwanese women. Journal of the Formosan Medical Association, 107(2):129-38.

8. Ahmed Y, van Iddekinge B, Paul $\mathrm{C}$ et al. (1993): Retrospective analysis of platelet numbers and volumes in normal pregnancy and in pre- eclampsia. BJOG: An International Journal of Obstetrics \& Gynaecology, 100 (3):216-20.

9. Järemo P, Lindahl T, Lennmarken C et al. (2000): The use of platelet density and volume measurements to estimate the severity of pre- eclampsia. European Journal of Clinical Investigation, 30(12):1113-8.

10. Makuyana D, Mahomed K, Shukusho F et al. (2002): Liver and kidney function tests in normal and pre- 
eclamptic gestation: a comparison with non-gestational reference values. Cent AfrJ Med., 48 (5/6):55-9.

11. El Sherbiny W, Kamal S (2009): Circulating microparticles in pre-23 eclampsia. Anatolian Journal of Obstetrics \& Gynecology, 4:1-5.

12. El-garhey I, Mohammed A, Ismail A (2018): Role of mean platelet volume in prediction of preeclampsia. The Egyptian Journal of Hospital Medicine, 73 (10):7834-41.

13. Fay R, Hughes A, Farron $\mathbf{N}$ et al. (1983): Platelets in pregnancy: hyperdestruction in pregnancy. Obstetric Anesthesia Digest., 3 (4):103.

14. Eser A, Zulfikaroglu E, Eserdag S et al. (2011): Predictive value of middle cerebral artery to uterine artery pulsatility index ratio in preeclampsia. Archives of Gynecology and Obstetrics, 284 (2):307-11.

15. Mallikarjunappa B, Harish H, Ashish S et al. (2013): Doppler changes in pre-eclampsia. JIMSA., 26(4):21516.

16. Li H, Gudnason H, Olofsson $P$ et al. (2005): Increased uterine artery vascular impedance is related to adverse outcome of pregnancy but is present in only one- third of late third- trimester pre- eclamptic women. Ultrasound in Obstetrics and Gynecology: The Official Journal of the International Society of Ultrasound in Obstetrics and Gynecology, 25(5):459-63.

17. Özeren M, Dinç H, Ekmen Ü et al. (1999): Umbilical and middle cerebral artery Doppler indices in patients with preeclampsia. European Journal of Obstetrics \& Gynecology and Reproductive Biology, 82 (1):11-6.

18. Abdelrazik A, Mohammed T, Mohammed E (2020): The value of middle cerebral to umbilical artery Doppler ratio in the prediction of neonatal outcome among women with pregnancy induced hypertension. Al-Azhar Medical Journal, 49 (4):1713-22.

19. Brar H, Horenstein J, Medearis A et al. (1989): Cerebral, umbilical, and uterine resistance using Doppler velocimetry in postterm pregnancy. Journal of Ultrasound in Medicine, 8(4):187-91.

20. Arduini D, Rizzo G (1992): Prediction of fetal outcome in small for gestational age fetuses: comparison of
Doppler measurements obtained from different fetal vessels. J Perinat Med., 20:29-38.

21. Ghosh R, Oza H, Meel S (2017): Third trimester Doppler ultrasound as prediction of obstetric outcome in high-risk pregnancy, Gujarat, India. International Journal of Reproduction, Contraception, Obstetrics and Gynecology, 6(8):3519-23.

22. Shahinaj R, Manoku N, Kroi E et al. (2010): The value of the middle cerebral to umbilical artery Doppler ratio in the prediction of neonatal outcome in patient with preeclampsia and gestational hypertension. Journal of Prenatal Medicine, 4 (2):17-21.

23. Gramellini D, Folli M, Raboni S et al. (1992): Cerebral umbilical Doppler ratio as a predictor of adverse perinatal outcome. Obstet Gynecol., 79:416-20.

24. Ayaz A, Muhammad T, Hussain S et al. (2009): Neonatal outcome in pre-eclamptic patients. Journal of Ayub Medical College Abbottabad., 21 (2):53-5.

25. Brown M, Hague W, Higgins $J$ et al. (2000): The detection, investigation and management of hypertension in pregnancy: full consensus statement. Australian and New Zealand Journal of Obstetrics and Gynaecology, 40 (2):139-55.

26. El-Sokkary M, Omran M, Ahmed H (2011): Ratio of middle cerebral artery/umbilical artery Doppler velocimetry and status of newborn in postterm pregnancy. Br Med J., 280:282-3.

27. Yalti S, Oral Ö, Gürbüz B et al. (2004): Ratio of middle cerebral to umbilical artery blood velocity in preeclamptic \& hypertensive women in the prediction of poor perinatal outcome. Indian Journal of Medical Research, 120 (1):44-48.

28. Walsh S (2006): What causes endothelial cell activation in preeclamptic women?. The American Journal of Pathology, 169 (4):1104-8.

29. Shwarzman P, Waintraub A, Frieger $\mathrm{M}$ et al. (2013):Third- trimester abnormal uterine artery Doppler findings are associated with adverse pregnancy outcomes. Journal of Ultrasound in Medicine, 32(12):2107-13. 